Disponível em

http://www.anpad.org.br/rac

RAC, Rio de Janeiro, v. 18, Edição Especial, art. 2, pp. 22-40, Dezembro 2014

http://dx.doi.org/10.1590/1982-7849rac20141249

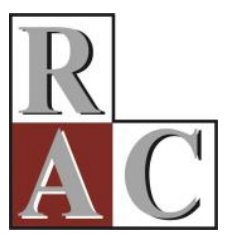

$(c)$ EY-NO

\title{
Formulação e Execução de Estratégias Políticas no Setor de Etanol: Um Modelo Processual
}

\section{Political Strategy Formulation and Implementation in the Ethanol Industry: A Procedural Model}

\author{
Antonio Thiago Benedete da Silva \\ E-mail: atbenedete@usp.br \\ Universidade Nove de Julho - UNINOVE \\ Rua Guaranésia, 425, 02112-000, São Paulo, SP, Brasil. \\ Carlos Afonso Caldeira \\ E-mail: carlos_caldeira@yahoo.com \\ Instituto de Ensino e Pesquisa; Fundação Getulio Vargas - EAESP/FGV \\ Rua Quatá, 300, 04546-042, São Paulo, SP, Brasil. \\ Rodrigo Bandeira-de-Mello \\ E-mail: rodrigo.bandeira.demello@fgv.br \\ Fundação Getulio Vargas - EAESP/FGV \\ Rua Itapeva, 474, 01332-000, São Paulo, SP, Brasil.
}

Artigo recebido em 12.12.2012. Última versão recebida em 16.05.2014. Aprovado em 23.05.2014. Publicado online em 29.10.2014. 


\title{
Resumo
}

Conquanto a importância das estratégias políticas para a sobrevivência e desempenho das organizações, parcos são os estudos que focam efetivamente os processos que as empresas utilizam no que concerne tais estratégias. Sob essa ótica que foi conduzido este estudo qualitativo com análise de conteúdo por categorização, para tanto, utilizando-se dados de entrevistas com tomadores de decisão sobre estratégias políticas em empresas de etanol, bem como dados secundários sobre o setor e a atuação política das empresas analisadas. Com base nos dados, este artigo sugere um modelo para o processo de formulação e execução de estratégias políticas, discutindo, em termos teóricos, as especificidades desse processo. Entre as peculiaridades do processo de formulação e execução de estratégias políticas estão volume de atividades realizadas no âmbito externo à firma, a prevalência de ações coletivas e a complexidade em apurar os resultados da implementação das estratégias políticas.

Palavras-chave: estratégias políticas corporativas; etanol; processo estratégico; estratégias políticas.

\begin{abstract}
Despite the importance of political strategies for firm survival and performance, there are few studies that focus on what is effectively the process that companies use to formulate and execute these types of strategies. We have conducted a qualitative study, using content analysis through categorization to analyze data from interviews with political-strategy decision makers from Brazilian Ethanol companies, as well as secondary data on the sector. Based on the data, this article suggests a model for the process of formulating and implementing political strategy, discussing the details of this process in theoretical terms. Among the peculiarities of the political strategy process, the most important are the amount of activities conducted outside the firm, the prevalence of collective action and the complexity of evaluating political strategy results.
\end{abstract}

Key words: corporate political activities; ethanol; strategy process, political strategy. 


\section{Introdução}

As estratégias de não mercado, entre elas, as políticas, vêm sendo discutidas no domínio da estratégia empresarial desde a década de 1990 (Shaffer, 1995). Diversos autores concordam com a importância de tais tipos de ações para a sobrevivência e diferencial em termos de desempenho que tais estratégias políticas podem proporcionar (Lux, Crook, \& Woehr, 2011).

O desenvolvimento da literatura em estratégias políticas tem utilizado, primordialmente, conjuntos de firmas como unidade de análise, principalmente por uma questão de disponibilidade de dados, em especial, dados pertinentes a doações de campanha e dados de despesas em atividades de lobbying (que são públicos nos EUA) (Hillman, Schuler, \& Keim, 2004; Shaffer, 1995). Entretanto raríssimos estudos se voltam para, como assinalam Shaffer e Hillman (2000), a parte muito importante do entendimento do fenômeno: como as decisões são tomadas e como estas são implementadas. Entender tais detalhes processuais ajuda a compreender o fenômeno de duas maneiras. No primeiro caso, a partir do entendimento do processo de formulação, pode-se dar (mais) luz a resultados hoje contraditórios sobre o fenômeno. A maioria dos artigos que estuda a relação entre estratégias políticas e desempenho encontra pouca ou nenhuma relação (Hillman et al., 2004; Lux et al., 2011). No segundo caso, ao entender a natureza da formulação e execução das estratégias políticas, pode-se estudar se este processo é fundamentalmente semelhante ao processo de formulação e execução estratégica (Hutzschenreuter \& Kleindienst, 2006; Mintzberg, 1978; Pettigrew, 1992). A maioria dos estudos em estratégias políticas, implicitamente, leva esta semelhança em consideração, mesmo que os detalhes não tenham sido estudados e comparados.

Como laboratório para levantamento de dados, foi escolhido o setor de produção sucroalcooleiro brasileiro. O Brasil tem sido um dos maiores produtores mundiais de cana-de-açúcar no decurso da história. O setor foi escolhido porque a interação do setor e governo é antiga. É possível argumentar que a própria existência do setor deve-se a uma ação governamental nos idos de 1500. Ao longo desta trajetória, diversas vezes, o setor teve negociações e benefícios oferecidos pelo governo (séculos XVI, XVIII, XIX e, especialmente, no século XX) (Leão, 2002; Szmrecsanyi \& Moreira, 1991). A escolha do setor também se justifica por uma questão de relevância. O setor brasileiro tem importância mundial, alavancada pelo advento do carro Flex e por se mostrar como uma alternativa viável aos combustíveis fósseis nos anos 2000 (Amatucci \& Spers, 2010). A escolha do setor também se justifica por motivos de propensão à atividade política na época do estudo. A partir de 2008, o setor entrou em grande crise, causada tanto pelos efeitos da crise mundial como pelas políticas de preços de combustíveis do governo federal brasileiro (Neves \& Conejero, 2010).

Este artigo contribui para o corpo teórico de estratégias políticas de duas maneiras. Primeiramente, ao descrever o processo de formulação e execução de estratégias políticas. Também por descrever as idiossincrasias do processo de formulação e execução de estratégias políticas em relação aos conceitos do processo estratégico (termo em inglês, strategy-process).

\section{Referencial Teórico}

\section{Estratégias políticas}

A definição de estratégias envolve um conjunto de decisões sobre como a organização irá interagir com o ambiente externo, responder às suas demandas e pressões e também tentar influenciá-lo quando a organização pretende adotar uma postura antecipatória em relação à dinâmica da evolução de variáveis mercadológicas e não mercadológicas (Hillman et al., 2004). Tradicionalmente, a literatura em estratégia foca a organização e suas estratégias de mercado (Baron, 1995). Entretanto, o desempenho das empresas pode ser afetado por componentes externos não mercadológicos - notadamente o governo e a definição das regras institucionais e legais e o sistema judiciário. Firmas têm a necessidade de 
desenvolver estratégias não mercadológicas que devem ser integradas às estratégias mercadológicas, conforme afirma Baron (1995). A mais usada das estratégias de não mercado é a das estratégias políticas ou Corporate Political Actions (CPA) (Getz, 1997). Neste trabalho, define-se as estratégias políticas como as estratégias e ações voltadas a influenciarem as políticas públicas e outras regulamentações em favor da firma (Hillman et al., 2004).

O estudo das estratégias políticas tem se pautado em utilizar a firma ou setor como unidade de análise (Shaffer \& Hillman, 2000). Neste tipo de unidade macro, o avanço foi considerável no entendimento dos fatores antecedentes ao engajamento em estratégias políticas. Fatores relacionados à firma (tamanho, origem do capital) e à estrutura do setor (concentração) são significativamente relacionados ao engajamento em atividades políticas (Hillman et al., 2004). Estudos também tentam explicar a relação entre atividades e desempenho político ou econômico das empresas. Enquanto poucos conseguem estabelecer esta relação claramente (Hadani \& Schuler, 2013), a maioria dos estudos encontrou pouca relação entre estratégias políticas e desempenho (Ansolabehere, Figueiredo, \& Snyder, 2003; Lux et al., 2011). Entretanto pouco foco tem sido dado ao processo de escolhas e execução das estratégias políticas, bem como às questões organizacionais relativas a esta atividade. Hillman e colegas são específicos ao recomendarem mais estudos neste sentido (Hillman et al., 2004).

Ironicamente, apesar das estratégias políticas serem partes das estratégias de não mercado, o framework dominante para explicação das atividades políticas está focado em um mercado de compra e venda de políticas públicas (Bonardi, Hillman, \& Keim, 2005). Porém a falha em se demonstrar a relação entre investimento e retorno das estratégias políticas abre a oportunidade para que outras explicações não centradas em racionalidade econômica sejam exploradas para caracterizar as estratégias políticas (Ansolabehere et al., 2003). Parte destas explicações alternativas está voltada para as influências dos agentes (estrategistas políticos) no processo, influências estas que podem ser comportamentais (Hillman et al., 2004) ou cognitivas (Cook \& Barry, 1995).

Enquanto a formulação das estratégias políticas encontra amparo em poucos trabalhos (Cook \& Barry, 1995; Shaffer \& Hillman, 2000), as escolhas relativas à execução são geralmente classificadas de acordo com o modelo Hillman e Hitt (1999), que definem três tipos genéricos de estratégias políticas: estratégias de informação, estratégias financeiras e estratégias de construção de base de suporte, como mostra a Tabela 1.

Tabela 1

\section{Taxonomia das Estratégias Políticas}

\begin{tabular}{|c|c|c|}
\hline Estratégia & Tática & Características \\
\hline \multirow{4}{*}{$\begin{array}{l}\text { Estratégias de } \\
\text { informação }\end{array}$} & Lobby & \multirow{4}{*}{$\begin{array}{l}\text { Almejam atingir o tomador } \\
\text { de decisão em políticas } \\
\text { públicas através do } \\
\text { fornecimento de informação. }\end{array}$} \\
\hline & Financiamento de projetos de pesquisa. & \\
\hline & $\begin{array}{l}\text { Depoimentos com especialistas em determinado assunto } \\
\text { ou issue (questão política). }\end{array}$ & \\
\hline & Fornecimento de relatórios técnicos ou de opinião. & \\
\hline \multirow{4}{*}{$\begin{array}{l}\text { Estratégias de } \\
\text { incentivo financeiro }\end{array}$} & Contribuições para políticos ou partidos & \multirow{4}{*}{$\begin{array}{l}\text { Almejam atingir o tomador } \\
\text { de decisão em políticas } \\
\text { públicas através do } \\
\text { fornecimento de incentivos } \\
\text { financeiros. }\end{array}$} \\
\hline & Honorários por palestras. & \\
\hline & Pagamento de viagens e convenções. & \\
\hline & $\begin{array}{l}\text { Contratação de pessoas com experiência política e/ou ter } \\
\text { membros da firma concorrendo a cargos públicos. }\end{array}$ & \\
\hline
\end{tabular}




\section{Tabela 1 (continuação)}

\begin{tabular}{lll}
\hline Estratégia & Tática & Características \\
\hline $\begin{array}{l}\text { Estratégias de } \\
\text { construção de base } \\
\text { de suporte }\end{array}$ & Mobilização da base de empregados, fornecedores, etc. & $\begin{array}{l}\text { Almejam atingir o tomador } \\
\text { de decisão em políticas } \\
\text { públicas indiretamente, } \\
\text { através de pressão da }\end{array}$ \\
& Propaganda política. & $\begin{array}{l}\text { opinião pública e base de } \\
\text { eleitores. }\end{array}$ \\
& Conferências públicas. & \\
& Programas de educação política. & \\
\hline
\end{tabular}

Nota. Fonte: Traduzido pelos autores, de Hillman, A. J., \& Hitt, M. A. (1999). Corporate political strategy formulation: a model of approach, participation, and strategy decisions. Academy of Management Review, 24(4), 825-842. doi: 10.5465/AMR.1999.2553256

A contribuição desse corpo teórico para explicar a vantagem competitiva das empresas que operam no Brasil é, no entanto, limitada, se importada sem as devidas adaptações. Primeiro, a consideração de mercados competitivos que transacionam políticas públicas não encontra aderência ao cenário nacional no qual as firmas buscam favores individuais por meio de relações pessoais. Segundo, o ponto de vista considerado nos estudos internacionais é preferencialmente o das firmas de países desenvolvidos ou multinacionais que se instalam em países emergentes. Explicações sobre como firmas locais lidam com o governo local e como essa relação pode beneficiá-las estão em desenvolvimento (Bandeira-de-Mello, Marcon, \& Alberton, 2008; Brey, Camilo, Marcon, \& Bandeira-de-Mello, 2013; Costa, Bandeira-de-Mello, \& Marcon, 2013; Fernandes, Bandeira-de-Mello, \& Zanni, 2012; Lazzarini, 2011; Samuels, 2001). Apesar do avanço democrático e do fortalecimento das instituições de mercado, como os intermediários financeiros, os mercados de capitais e os marcos regulatórios, o ambiente político das firmas no Brasil continua exercendo um papel importante nas decisões estratégicas (Bandeira-de-Mello \& Marcon, 2005).

\section{Processo estratégico}

Os processos de formulação e execução estratégica estão no cerne do desenvolvimento do campo da estratégia (Hutzschenreuter \& Kleindienst, 2006), apesar da recente tendência do campo em tornarse uma ciência mais próxima à economia, utilizando unidades de análise mais agregadas (Shaffer \& Hillman, 2000). Trabalhos clássicos, como os de Mintzberg (1978), Quinn (Mintzberg \& Quinn, 1998) e Van De Ven (1992), formam as bases conceituais desta corrente.

A popularidade deste assunto de pesquisa resultou num largo campo considerado fragmentado (Hutzschenreuter \& Kleindienst, 2006), com diversas correntes de discussão específicas, entre elas, a discussão do sentido do nascimento das iniciativas estratégicas (Burgelman, 1983; Mintzberg, 1978; Noda \& Bower, 1996) ou, ainda, o conjunto de discussões a respeito dos modos de pesquisa sobre processo estratégico (Pettigrew, 1992; Van De Ven, 1992).

Conquanto tal dispersão é possível descrever um modelo geral para o fenômeno (Hutzschenreuter \& Kleindienst, 2006). Os antecedentes à formulação e execução estão ligados a características de contexto (complexidade, posição estratégica) e características da firma (tamanho, rotinas e nível de desempenho passado). O processo estratégico (formulação e execução) em si é permeado por características pessoais e cognitivas dos estrategistas e por questões ligadas ao issue estratégico (neste trabalho, utiliza-se o termo em inglês issue, por que a palavra em português, questão, não tem o mesmo significado). Por fim, os resultados (outcomes) do processo afetam as vantagens competitivas e retroalimentam as categorias antecedentes (Hutzschenreuter \& Kleindienst, 2006). 


\section{Procedimentos Metodológicos}

Nesta seção serão apresentados os procedimentos metodológicos utilizados para atingir os objetivos do trabalho, envolvendo a caracterização do tipo de pesquisa, a natureza e coleta dos dados, bem como a técnica utilizada para a análise dos dados.

\section{Tipo de pesquisa}

O objetivo desta pesquisa é investigar os processos pelos quais as estratégias políticas de empresas do setor de etanol são formuladas e executadas. Por se tratar de um tema pouco estudado na academia brasileira de administração e o referencial conceitual ser majoritariamente internacional e construído a partir de realidades diversas do caso brasileiro, foi conduzido um estudo descritivo-analítico e de natureza qualitativa.

Segundo Cooper e Schindler (2003), a pesquisa descritiva tem por finalidade descrever fenômenos ou características associados com a população-alvo, estimar proporções de uma população que tenha essas características e descobrir associações entre as variáveis. Yin (2008) aponta que o estudo de casos é uma escolha de metodologia adequada quando a questão de pesquisa tenta responder a questões do tipo como ou por que. A estratégia de estudo de casos também pode ser uma escolha quando o objetivo não é simplesmente a descrição, mas a geração (Eisenhardt, 1989) ou complementação de teorias (Siggelkow, 2007).

Segundo Richardson (2009), a abordagem qualitativa difere, em princípio, da quantitativa à medida que não emprega um instrumental estatístico como base do processo de análise de um problema. Não pretende numerar ou medir unidades ou categorias homogêneas. Para o autor, a abordagem qualitativa de um problema justifica-se por ser uma forma adequada para entender a natureza de um fenômeno social. Em princípio, as investigações que se voltam para uma análise qualitativa têm como objeto situações complexas ou estritamente particulares. Os estudos que empregam uma metodologia qualitativa podem descrever a complexidade de um determinado problema. Merriam (1988) destaca o caráter particularista, descritivo e heurístico de um estudo de casos. Conforme Merriam, o estudo de caso deve estar centrado em uma situação ou evento particular, bem delimitado, e que deve ser relevante para o objetivo da pesquisa. Deve ser descritivo tanto na obtenção dos dados como no relatório de resultados.

Finalmente, Pettigrew (1992), em suas considerações sobre as pesquisas de campo voltadas ao estudo do processo estratégico, destaca algumas peculiaridades que acredita serem importantes para a condução de pesquisas cuja unidade de análise é processo: a necessidade de análise em vários níveis processuais ou etapas, a importância do contexto e da ação no estudo de processos e a necessidade de ligar a análise do processo com a análise dos seus resultados (outcomes).

\section{Técnica de coleta de dados}

Nesta pesquisa, foram trianguladas múltiplas fontes de dados para investigar o fenômeno das estratégias políticas; foram usados tanto dados de natureza primária quanto secundária. Os dados primários foram coletados por meio de entrevistas que seguiram protocolos com questões abertas e aplicadas a informantes que atuam no setor de etanol. Como técnica de coleta de dados, a entrevista é bastante adequada para a obtenção de informações acerca do que as pessoas sabem, creem, esperam, sentem ou desejam, pretendem fazer, fazem ou fizeram, bem como acerca de suas explicações ou razões a respeito de aspectos precedentes (Selltiz, Wrightsman, \& Cook, 1976). Foram realizadas 15 entrevistas envolvendo pesquisadores, gestores, empresários do setor e membros da diretoria de associações de classe de defesa dos interesses do etanol.

Como colocam Hillman, Schuler e Keim (2004), a estratégia política é um jogo praticado quase que exclusivamente pelos altos escalões e times de liderança nas empresas. E a seleção dos entrevistados 
levou essa característica em questão e, por isso, foram selecionados apenas entrevistados de altos níveis hierárquicos das empresas (presidentes, diretores e sócios-fundadores), bem como se certificou de que os respondentes eram (ou foram) responsáveis por decisões sobre as estratégias políticas. As empresas representadas pelos entrevistados detinham aproximadamente um sexto da capacidade de moagem de cana brasileira, à época das entrevistas. Os pesquisadores não tinham qualquer vínculo com os entrevistados, que foram contatados utilizando-se a base de contatos de centro de estudos de agronegócios de uma das escolas vinculadas aos pesquisadores (nenhum dos pesquisadores pertence ao centro). Entrevistados também foram contatados através da indicação de outros entrevistados. Antes de ir a campo colher as entrevistas dos tomadores de decisão, foram realizadas entrevistas com especialistas do setor. Estas entrevistas serviram para balizar o conhecimento adquirido com o material público sobre o setor (Eisenhardt, 1989). As entrevistas foram realizadas entre maio de 2011 e janeiro de 2012 e foram conduzidas em conjunto pelos pesquisadores autores deste trabalho. Cada entrevista durou aproximadamente 90 minutos. Para viabilizar a coleta dos dados primários, foi garantida aos informantes a confidencialidade de suas identidades. Os entrevistados foram, então, designados de 1 a 15 (Tabela 2). Quando foi permitida a gravação das entrevistas, esta foi executada e, depois, transcrita. Quando a gravação não foi possível (na maioria das vezes, por questões de confidencialidade), utilizou-se de notas de campo o mais detalhadas possível (Emerson, Fretz, \& Shaw, 1995). Além disso, as notas eram repassadas e revisadas em curto espaço de tempo, após as entrevistas (Eisenhardt, 1989). A Tabela 2 descreve as características dos entrevistados.

Tabela 2

\section{Características dos Entrevistados}

\begin{tabular}{|c|c|}
\hline Denominação do entrevistado & Descrição \\
\hline Entrevistado 1 & $\begin{array}{l}\text { Pesquisador e professor universitário atuante em temas relacionados ao setor } \\
\text { de etanol. }\end{array}$ \\
\hline Entrevistado 2 & Consultor especialista no setor de etanol. \\
\hline Entrevistado 3 & $\begin{array}{l}\text { Pesquisador, professor e ex-membro da equipe ministerial da pasta agrícola } \\
\text { em governos anteriores. }\end{array}$ \\
\hline Entrevistado 4 & Sócio-diretor de usina de álcool e açúcar localizada no Paraná. \\
\hline Entrevistado 5 & $\begin{array}{l}\text { Diretor agrícola de usina de álcool e açúcar localizada no Paraná. Foi } \\
\text { responsável por conduzir negociação com o governo estadual para a } \\
\text { implantação de projeto de infraestrutura de interesse dos usineiros do estado. }\end{array}$ \\
\hline Entrevistado 6 & $\begin{array}{l}\text { Diretor de relações institucionais de empresa do agronegócio multinacional } \\
\text { estrangeira que diversificou os negócios para o setor de etanol. }\end{array}$ \\
\hline Entrevistado 7 & $\begin{array}{l}\text { Diretor executivo de associação de classe que representa os interesses de um } \\
\text { grupo de empresas do setor de etanol. }\end{array}$ \\
\hline Entrevistado 8 & $\begin{array}{l}\text { Diretor de relações institucionais de usina de álcool, açúcar e produção de } \\
\text { energia elétrica controlada por um grande grupo empresarial de origem } \\
\text { nacional. }\end{array}$ \\
\hline Entrevistado 9 & $\begin{array}{l}\text { Presidente de associação de classe que representa os interesses de um grupo } \\
\text { de empresas do setor de etanol. }\end{array}$ \\
\hline Entrevistado 10 & Sócio de usina de localizada no estado de São Paulo. \\
\hline Entrevistado 11 & Sócio de grupo nacional proprietário de usinas de açúcar e álcool. \\
\hline Entrevistado 12 & Diretor financeiro de um grupo de usinas de etanol e açúcar. \\
\hline
\end{tabular}




\section{Tabela 2 (continuação)}

\begin{tabular}{ll}
\hline Denominação do entrevistado & Descrição \\
\hline Entrevistado 13 & $\begin{array}{l}\text { Diretor de relações institucionais de usina de álcool e açúcar pertencente a } \\
\text { grupo diversificado estrangeiro. }\end{array}$ \\
Entrevistado 14 & $\begin{array}{l}\text { Diretor executivo de empresa que intermedia transações comerciais no setor } \\
\text { de etanol e presidente de associação internacional voltada ao comércio } \\
\text { internacional de etanol. }\end{array}$ \\
Entrevistado 15 & $\begin{array}{l}\text { Presidente de usina de álcool e açúcar, diretor de associação de classe que } \\
\text { representa os interesses do setor e membro do conselho de cooperativa de } \\
\text { usinas de açúcar e álcool. }\end{array}$ \\
\hline
\end{tabular}

As entrevistas abordaram questões sobre as estratégias políticas usadas no setor de etanol e quanto aos processos de formulação e execução das estratégias em linha com o referencial teórico (Getz, 1997; Hillman et al., 2004; Hillman \& Hitt, 1999). Dados secundários foram obtidos em jornais, revistas, anais de congressos, vídeos e na base de dados do site Transparência Brasil. Entre artigos acadêmicos, artigos de jornal, livros, tabelas de dados e apresentações de congressos, foram utilizadas dezenas de fontes para o entendimento do setor. Os dados secundários desempenharam um papel importante nesta pesquisa, pois ajudaram a complementar os dados fornecidos pelos informantes e buscaram evidências adicionais do fenômeno das estratégias políticas no setor de etanol.

\section{Técnica de análise dos dados}

As categorias de análise foram definidas baseando-se no referencial teórico (Getz, 1997; Hillman et al., 2004; Hillman \& Hitt, 1999; Hutzschenreuter \& Kleindienst, 2006). Para categorizar as escolhas sobre estratégias políticas, usou-se a classificação de Hillman e Hitt (1999). Os dados foram analisados concomitantemente com as entrevistas, seguindo recomendações de Eisenhardt (1989) e Pettigrew (1992). Executar as análises desta maneira permite que se façam os ajustes necessários, entre os quais, aqueles atinentes à coleta de dados (Eisenhardt, 1989).

Utilizou-se a análise de conteúdo clássica com categorização (Bauer, 2000) como técnica para a análise dos dados. A análise de conteúdo torna possível o aprofundamento nas questões de significados de ações e interações humanas, o que permite análises mais profundas e de fatos pouco perceptíveis a partir dos métodos qualitativos. É, assim, possível que se obtenha inferências além do manifesto, inclusive sobre os conteúdos que os entrevistados deixaram subtendidos (H. Freitas \& Janissek, 2000). O referencial de codificação foi desenvolvido para se ajustar tanto às considerações teóricas como aos materiais colhidos (Bauer, 2000).

\section{Apresentação e Análise dos Resultados}

\section{Etapas do processo estratégico político}

Usando as classificações típicas de processo estratégico (Hutzschenreuter \& Kleindienst, 2006; Pettigrew, 1992), é possível referir que o processo estratégico político tem, de maneira central, as mesmas características. A estrutura do processo estratégico em si se mostrou semelhante ao do processo estratégico tradicional. O ponto de maior similaridade é, naturalmente, a existência de uma issue, tanto estratégia como política. Como evidenciam alguns autores, o processo de interpretação da issue estratégica é central ao processo de planejamento estratégico (Hutzschenreuter \& Kleindienst, 2006). Analogamente, a questão da issue política é central para o caso do processo de estratégia política (Bonardi \& Keim, 2005). A questão da issue central do setor de etanol, o controle de preços por parte do governo, foi bastante debatida nas entrevistas. Um dos entrevistados afirmou que 
“é a repetição da tentativa do governo em segurar a inflação em uma escala menor que nos traz problemas. A gasolina já deveria ter sido ajustada. Começa a misturar coisas entre a gestão publica e a privada" (E11).

Enquanto um dos produtores declarou "que com a situação política da gasolina ficou ainda mais difícil, nossa maior competição é a própria política do governo" (E12), outro complementou dizendo que "(tem de) colocar a gasolina no preço que tem de ser" (E15). Nesse sentido, ainda outro colocou que "não esquecendo que temos uma pressão de preços ..., precisamos ter preços de gasolina e etanol reais" (E5).

A questão dos antecedentes ao processo estratégico se fez presente nas entrevistas. Nos casos estudados, especialmente as questões antecedentes de desempenho e fatores antecedentes ligados a características dinâmicas organizacionais (Hutzschenreuter \& Kleindienst, 2006). Os antecedentes de desempenho do setor, que de maneira geral tem sofrido com as decisões governamentais de combater a inflação usando o preço da gasolina (J. Freitas, 2011) e, como consequência, defasando o preço do etanol, aparecem de maneira patente nas entrevistas. Um entrevistado declarou que:

“O BNDES se junta com os 10 maiores grupos, faz uma lista de quem poderia continuar e de quem deveria ser vendido. É por isso que vemos grandes famílias tradicionais com 60 anos de negócios sendo obrigadas a vender e entregar para multinacionais a preços não ideais" (E10).

Outro entrevistado afirmou que "houve a redução de 200 famílias no negócio para 60 famílias, e isso afeta a estratégia (política)" (E3). A dependência das ações do governo para o desempenho das empresas também foi assunto de praticamente todas as entrevistas. "Este negócio só funciona com a ajuda do governo" (E10), indicou outro entrevistado.

Outro ponto de bastante semelhança entre os processos de estratégia original e de estratégia política é a questão das influências cognitivas tanto nas decisões estratégicas (Porac, Thomas, \& BadenFuller, 2011) quanto nas decisões sobre estratégia política (Cook \& Barry, 1995). As afirmações dos grupos de produtores existentes, quanto às maneiras corretas de fazer estratégia política, foram claramente diferentes dependendo do grupo social do produtor. Produtores tradicionais estabelecidos no país argumentaram a favor de estratégias predominantemente coletivas, enquanto novos players de mercado não viram problema em atuar também de forma coletiva. Há uma espécie de monitoramento informal sobre tais iniciativas. Um entrevistado afirmou que "quando há uma tentativa de uma empresa conseguir benefícios individuais junto ao governo, o setor (grupo de empresas) se mobiliza para tentar boicotar" (E12). Produtores tradicionais afirmaram ainda que "A UNICA tem uma regra de que usineiros não vão mais para Brasília ... ANFAVEA era assim até pouco tempo" (E11). Outro entrevistado relatou que "ele não deve ir individualmente ... Ainda não se vai individualmente" (E14). Outro exemplo deste pensamento é que "o associativismo é a melhor maneira, porque funciona” (E10). Assim, aparecem claramente as maneiras certas de competir dentro de cada comunidade cognitiva (Porac et al., 2011). Estes filtros entre maneiras de competir afetaram as escolhas em termos de políticas estratégicas por parte dos entrevistados (chamar para ir individualmente a Brasília, por exemplo). Argumentando ao contrário, um dos executivos de empresa multinacional salientou: "não vejo problema em ir individualmente. Problemas comuns, vamos via associação; particulares, vamos sozinhos” (E4).

No tocante às escolhas de estratégias políticas (ou formulação), Hillman e Hitt (1999) definem três tipos genéricos de estratégias políticas: estratégias de informação, estratégias financeiras e estratégias de construção de base de suporte. Os dados levantados nesta pesquisa mostram que há a utilização dos três tipos de estratégias no setor de etanol. Dependendo do nível de governo que se pretenda atingir, há a preferência por determinado tipo de estratégia e tática.

Em âmbito estadual, a estratégia financeira é bastante empregada e a tática é a doação à campanha de Deputados Estaduais e Governador, embora haja doações a candidatos a Deputados Federais e Presidente da República. No Paraná, as usinas que participam da ALCOPAR realizaram doações a campanhas de candidatos nas eleições de 2002, 2006 e 2010, conforme lista-se na Tabela 3. 
Tabela 3

Valores Doados pelas Usinas Associadas à Alcopar

\begin{tabular}{lccc}
\hline Usina & $\mathbf{2 0 0 2}$ & $\mathbf{2 0 0 6}$ & $\mathbf{2 0 1 0}$ \\
\hline Usina Alto Alegre & $\mathrm{R} \$ 185.272,36$ & $\mathrm{R} \$ 90.000,00$ & $\mathrm{R} \$ 295.000,00$ \\
Cooperval & $\mathrm{R} \$ 2.000,00$ & $\mathrm{R} \$ 28.000,00$ & $\mathrm{R} \$ 10.400,00$ \\
Usina de Açúcar e Álcool Goioerê Ltda & $\mathrm{R} \$ 5.000,00$ & $\mathrm{R} \$ 15.000,00$ & - \\
Dasa - Destilaria Americana S/A & - & $\mathrm{R} \$ 71.000,00$ & - \\
$\begin{array}{l}\text { Copagra - Cooperativa Agroindustrial do } \\
\text { Noroeste Paranaense }\end{array}$ & - & $\mathrm{R} \$ 3.000,00$ & $\mathrm{R} \$ 1.100,00$ \\
Renuka Vale do Ivaí S/A & - & $\mathrm{R} \$ 58.000,00$ & - \\
$\begin{array}{l}\text { Coopcana - Cooperativa Agrícola Regional de } \\
\text { Produtores de Cana Ltda }\end{array}$ & - & - & $\mathrm{R} \$ 5.000,00$ \\
\hline
\end{tabular}

Nota. Fonte: elaborado pelos autores com dados do As Claras 2012, Transparência Brasil. (n.d.). Quem financia quem nas eleições. Recuperado de http://www.asclaras.org.br/

Especificamente, no caso das doações dos associados da ALCOPAR, o mecanismo pelo qual a doação traz resultados para as empresas é que, uma vez eleitos, os Deputados Estaduais auxiliam no agendamento de audiências públicas com o Governador. Uma demanda específica que está em curso é a construção de um alcoolduto que liga Maringá ao Porto de Paranaguá. Tal infraestrutura demanda investimentos consideráveis e as destilarias buscam no governo apoio para fazer frente às necessidades de capital. Como noticiado pelo Jornal Cana (2007), há evidências do mecanismo que relaciona doação a candidatos e acesso a governador:

A ALCOPAR solicitou ao governador Roberto Requião, durante reunião em 20 de março de 2007, a construção de um alcoolduto ligando Maringá, no Noroeste do Paraná, ao Porto de Paranaguá. Na percepção da Associação, a obra beneficiará uma região que é o centro da produção de açúcar e álcool no estado. Dessa reunião, foi solicitada pelo governador a realização de estudos técnicos aprofundados, em conjunto com o Porto de Paranaguá e a Companhia Paranaense de Energia (Copel) (Jornal Cana, 2007, p. 16).

Tais dados corroboram as afirmações de Baron (1995), no sentido de que o desempenho das estratégias de mercado pode ser afetado pela atuação de componentes externos não mercadológicos, portanto, havendo a necessidade de desenvolver estratégias integradas. Outro exemplo é o do perfil de doações a campanhas entre as esferas governamentais, que é diverso, considerando diferentes grupos de empresas, como sublinha o autor do estudo que compartilha dados com esta pesquisa (Caldeira, 2012). O setor de etanol, em 2010, doou mais de 54 milhões de reais, mas de maneira bem diversa se considerados os grupos de produtores (Tabela 4). 
Tabela 4

Doações por Cargo e Nível de Governo, Efetuadas pelas Empresas do Setor de Etanol (R\$ MM) Eleição 2010

\begin{tabular}{llllll}
\hline Cargos & $\begin{array}{l}\text { Produtores } \\
\text { tradicionais } \\
\text { associados à } \\
\text { UNICA }\end{array}$ & $\begin{array}{l}\text { Novos } \\
\text { entrantes } \\
\text { associados à } \\
\text { UNICA }\end{array}$ & $\begin{array}{l}\text { Produtores } \\
\text { tradicionais } \\
\text { não associados } \\
\text { à UNICA }\end{array}$ & Outros & Total Geral \\
\hline Federal & & & & 17,7 \\
\hline Presidente & 5,7 & 9,9 & 0,2 & 2,0 & 3,9 \\
Senador & 0,7 & 2,2 & 0,8 & 0,2 & 12,6 \\
Deputado Federal & 2,4 & 5,1 & 4,4 & 0,7 & 34,2 \\
Total Federal & 8,8 & 17,2 & 5,4 & 2,9 & 12,5 \\
\hline Estadual & & & & 1,8 & 7,2 \\
\hline Governador & 0,6 & 5,0 & 5,1 & 0,0 & 19,7 \\
Deputado Estadual & 1,7 & 2,3 & 3,2 & 1,8 & $\mathbf{5 3 , 9}$ \\
\hline Total Estadual & 2,3 & 7,3 & 8,3 & $\mathbf{4 , 6}$ & $\mathbf{1 3 , 4}$ \\
\hline Total Geral & $\mathbf{1 1 , 3}$ & $\mathbf{2 4 , 6}$ & & & \\
\hline
\end{tabular}

Nota. Fonte: Caldeira, C. A. (2012). Aspectos cognitivos da formulação de estratégias políticas: um estudo de caso no setor de etanol brasileiro (Dissertação de mestrado). Fundação Getulio Vargas, Escola de Administração de Empresas, São Paulo, $\mathrm{SP}$, Brasil.

Destaca-se que mais de uma estratégia pode ser usada ao mesmo tempo e de modo complementar. Como exemplificou um entrevistado (E2), foi utilizada a estratégia de informação e realizado um estudo técnico para avaliar a viabilidade do projeto de construção do alcoolduto e os benefícios que traria ao estado. Esse relatório direcionou as discussões e a negociação de apoio por parte do governo. Além disso, destaca-se o caráter coletivo da atuação sobre a issue. Conforme apontou ainda o mesmo entrevistado, "a atuação coletiva é mais fácil de ter resultados, pois temos menos pessoas sentando à mesa todos os meses para discutir o problema, fica mais fácil. Vinte pessoas são o setor” (E2).

Outro entrevistado declarou que a empresa investe em estratégia de informação, usando uma estrutura, apesar de reduzida, permanente em Brasília, com a designação de um funcionário e existência de escritório virtual (E5). Além disso, a empresa contrata uma consultoria. "Há um escritório de relações governamentais que nos atende. Ele faz o monitoramento do ambiente político, se preciso fazer contato, me dá o perfil do ministro e o telefone da secretária" (E5).

A estratégia de informação também é utilizada pela associação de classe, que representa um grupo de usinas do setor, em conjunto com a construção de bases de apoio:

“A forma mais importante é via o projeto de comunicação institucional e há participantes de vários elos da cadeia. As ações terão maiores impactos sobre a sociedade civil, conscientização de estudantes (mudanças climáticas, etanol como uma das possibilidades de solução), municípios canavieiros (mostrar a importância do setor), também trabalho junto a parlamentares. No ano passado, fizemos um evento levando especialistas para informar os legisladores e repetimos em seis assembleias estaduais" (E7).

Um dos depoimentos informa que há utilização de estratégias de informação em conjunto com as estratégias financeiras. Já em relação à legitimidade das estratégias financeiras, um entrevistado colocou: 
"Não vejo nenhum demérito em usar estratégias como apoio aos deputados federais e estaduais da região, prefeitos. O pagamento de viagens e convenções também acontece, tende a ser mais evitado devido aos escândalos recentes. Relações públicas e conferências são muito, muito usadas, bem mais utilizadas do que as outras" (E4).

Um dos entrevistados aponta que uma das razões para a utilização das estratégias de informação é que "o governo tem equipes de baixa preparação, deficiência estrutural do governo" (E14). O outro entrevistado, diretor de relações institucionais de um grupo de usinas pertencentes a um grupo empresarial diversificado, apontou que quando emprega estratégia financeira é o grupo empresarial que concentra a responsabilidade por ela (E8). Na percepção do entrevistado, a interlocução com governo federal se dá mais via atuação coletiva, principalmente via associação de classe. "O relacionamento local e federal tem o mesmo peso. Entretanto o federal ninguém conseguirá fazer sozinho e há concentração de ações por meio da associação" (E12), conforme informou um dos produtores.

O presidente de uma associação de classe que representa os interesses de um grupo de empresas do setor de etanol, a qual utiliza tanto a estratégia de informação como a estratégia de construção de base de apoio, relatou:

"Fazemos muitas ações de opinião pública e usamos relações públicas. Atendemos em média 30 jornalistas por dia e recebemos 180 delegações por ano. Existe ainda um programa permanente de apoio a estudos da academia e de centros de pesquisa. Temos também a atuação de lobby nos Estados Unidos e em Brasília. Nos EUA é muito mais bem-sucedido do que em Brasília, pois é mais fácil, mais organizado e transparente. Aqui no Brasil o lobby que se faz é de corredor. Nós adoraríamos que o lobby fosse regulamentado" (E9).

Os dados fornecidos pelos entrevistados mostram que a atuação nas associações de classe que representam o setor possibilita a coordenação de estratégias financeiras realizadas em conjunto pelas usinas.

"A associação de classe faz uma coordenação informal das doações a campanhas. Ela recebe a lista dos partidos e coordena a doação entre os sócios. É importante falar também que a doação não dá compromisso, apenas abre a porta. Outro ponto é que quando a doação é local, cada um faz o seu” (E11).

Um entrevistado enfatizou a importância da atuação coletiva das usinas, mesma opção utilizada pela empresa do outro produtor. "Em resumo, a atuação institucional é feita pelos sindicatos. Apesar do nosso estado ser pequeno, o sindicato aparece bastante. Feita dessa forma, a ação fica mais despersonalizada e mais representativa" (E12). Ainda para outro entrevistado, "a relação institucional é de suma importância, na medida em que há a necessidade definir o papel estratégico que o etanol terá na matriz energética brasileira nos próximos anos e definir regras institucionais claras para o setor. Entretanto a interlocução com o governo federal é problemática" (E3).

A questão da possibilidade de utilização de estratégias coletivas, menos comuns em estratégia competitiva, permite fazer uma grande separação entre os dois processos. Por exemplo, um dos entrevistados afirmou que o modo de ação da UNICA havia mudado, segundo ele, "os usineiros estavam proibidos de ir a Brasília (fazer lobbying)" (E11). Outro entrevistado referiu que "ele não deve ir individualmente ... Ainda não se vai individualmente" (E15). Outro exemplo deste pensamento é que "Associativismo é a melhor maneira, porque funciona." (E10). Ainda outro indicou que

"Isso não inviabiliza nossa participação via associação. "As associações das quais fazemos partes (e são muitas) têm também a sua agenda política. Temas que são comuns ao setor, nos alinhamos via associação e o protagonismo se dá via associação. Posso ir, liderado pela associação, acompanhar a demanda. Não vou agir em paralelo, competir ou me adiantar a UNICA. Se tivermos reuniões individuais, reportamos para a associação o ocorrido. Ou alinhamos agenda com a associação antes de uma reunião individual" (E13).

A representatividade das atividades coletivas evidência um competência que não parece tão importante em processos de planejamento estratégico, sendo relevada a estudos de fusões e aquisições e alianças estratégicas: as capacitações necessárias para executar estratégias em parceria (Doz, 1996; Hamel, 1991). Esta questão da terceirização de partes do processo de estratégia política de uma empresa parece ser uma das idiossincrasias mais importantes do processo de estratégia política. Tal terceirização 
pode ocorrer de maneira individual e no processo de monitoramento, por exemplo, ao contratar-se uma consultoria para fazer o monitoramento de legislação (Mancuso, 2004). Alguns depoimentos colhidos enfatizam que a complexidade das leis brasileiras faz com que esta atividade de leitura do ambiente (por exemplo, leitura dos diários oficiais diariamente) seja naturalmente contratada fora da empresa por questões de escala. Também, pode ocorrer de maneira individual através da contratação de consultorias especializadas em fazer lobbying, ou o acesso aos formadores de opinião políticos (Galan, 2012). Ainda mais específica é a possibilidade de terceirização do processo ou de parte do processo para as associações. Esta característica gera uma série de mudanças na dinâmica de formulação e execução da estratégia política. À época do estudo, existia grande embate pelas direções que a maior associação de interesse do setor, a UNICA, deveria tomar (Pereira \& Chade, 2012). Como bem colocou o clássico de Olson (1971), os problemas decorrentes da tomada de decisão em conjunto e da divisão dos ganhos de forma desproporcional incutem a necessidade de tomar decisões de maneira diferente: players dominantes devem ditar o tom, player pequenos devem fazer freeriding. Por exemplo, produtores mais antigos comentam que "a associação está acompanhando sobre o controle de terras em função de Bunge e Cosan" (E10). Outro afirmou que "as terras para estrangeiros não deveriam estar na associação, mas está (na associação) por pressão dos estrangeiros ..., a BUNGE está usando a associação para defender seus interesses na soja" (E11).

Finalmente, em termos de resultados (outcomes), o processo de estratégias políticas também tem diferenças em relação ao processo clássico. Um dos pontos ainda discutidos em relação às estratégias políticas está ligado à percepção de retorno. Como mencionado, as questões de relação entre uso de CPA e retorno financeiro são, ainda, inconclusivas (Lux et al., 2011). Neste sentido, depoimentos revelam a dificuldade de avaliação das estratégias políticas. Entre eles, o depoimento que aponta que "por isso uma vez por ano nós damos dinheiro para um monte de gente ... mas depois não conseguimos capitalizar" (E11). Estas questões das incertezas dos retornos são exacerbadas pela terceirização das atividades e pela utilização de estratégias coletivas de atuação. Um dos entrevistados comenta que "a associação parece um grande teatro, são todos atores" (E5). É exatamente a conclusão a que chegaram Cook e Barry (1995), que reportam o processo de negociação entre empresas e governos como uma série de representações de papéis entre as partes, sem que estas pareçam muito conscientes de qual papel estão desempenhando naquele momento, nem de que estes papéis mudam. Estes autores colocam que a importância das questões cognitivas e de outros modelos de simplificação da realidade é, neste contexto, ainda mais importante.

\section{Discussão}

A partir das evidências coletadas durante a pesquisa, desenvolveu-se o modelo de formulação e execução das estratégias políticas apresentado na Figura 1.



Figura 1. Modelo de Formulação e Execução da Estratégia Política. 
O processo de estratégias políticas assemelha-se ao dito processo tradicional em diversas esferas. A questão do contexto (Pettigrew, 1992) aparece como antecedente e influencia nas decisões. Por exemplo, a maioria dos entrevistados de empresas multinacionais, reporta que as doações de campanha são proibidas em medida de superproteção às pesadas sansões do Foreign Corrupt Practices Act. Esta seria uma evidência do contexto organizacional ou mesmo do contexto ambiental influindo no processo. A condição financeira dos produtores, após a crise de 2008, é outro exemplo de contexto influenciando o processo, desta vez, do contexto do desempenho (Pettigrew, 1992).

A etapa de formulação tem, também, muitas semelhanças com o processo tradicional. De acordo com nossos dados, existem as etapas de leitura (scanning) do ambiente para issues importantes. Encontrou-se muito vivamente a questão da interpretação dos issues e as influências pessoais dos gestores nesta implementação, como reportado em Caldeira (2012). Em termos das escolhas estratégicas, as evidências mostraram que o modelo de Hillman e Hitt (1999) foi capaz de capturar as decisões.

As etapas de implementação e acompanhamento de resultado ficaram marcadas pelas diferenças com o processo tradicional. Em primeiro lugar, a questão das dimensões internas e externas à firma, que perpassa quase todo o processo. Tanto a formulação como a implementação podem acontecer no ambiente externo, através de subcontratação (consultoria ou até mesmo associação). Conquanto estas possibilidades existam para o processo estratégico tradicional (Hamel, 1991), é possível argumentar que a parte realizada externamente no processo de estratégias políticas é muito mais evidente. As evidências mostraram que um dos reflexos desta característica no processo de estratégias políticas parece influenciar a visibilidade dos resultados das mesmas, com evidentes reflexos no próximo processo de planejamento das estratégicas políticas. Ademais, embora não tenham sido colhidas evidências de questões envolvendo o problema de confiança (Gulati, Lavie, \& Singh, 2009; Prashant \& Harbir, 2009), é possível supor que esta seja uma questão preponderante no processo de estratégias políticas, em função do componente externo à firma. Desta maneira, alguns mecanismos de aumento de confiança análogos aos dos propostos para alianças estratégicas, como o desenvolvimento de capacitações específicas (Doh, Lawton, \& Rajwani, 2012; Prashant \& Harbir, 2009), possivelmente, devem ser observados no processo de estratégias políticas.

Outra diferença que parece fundamental entre o processo estratégico tradicional e o de estratégias políticas reside no fato de que estratégias políticas são comumente executadas em contexto coletivo (Conejero, 2011), através de associações e coalizões (Schneider, 2010). As questões de cooperação receberam alguma atenção no âmbito dos estudos organizacionais e estudos de estratégia (Nielsen, 1988). No contexto das estratégias políticas, devido à sua importância e abundância, as questões das estratégias coletivas receberam menor atenção do que deveriam (Hillman et al., 2004). Como ilustração a este tipo de decisão e sua importância, recentemente, os autores tiveram acesso a uma pesquisa realizada com grandes empresas brasileiras (cerca de 30) sobre suas áreas de gestão governamental. Na média, as empresas desta amostra participavam de 14 associações de classe.

Relacionado ao comentário anterior, existe a questão da pouca clareza na interpretação dos resultados das estratégias políticas. A incerteza em relação aos desempenhos e retornos políticos e financeiros das estratégias políticas é uma questão há muito debatida (Ansolabehere et al., 2003). Se esta é uma decisão de investimento, e o investimento é positivo, por que, em geral, as empresas doam menos do que a legislação permite? Parte da explicação pode estar na dificuldade de saber os retornos deste processo. As possibilidades de estratégias são inúmeras, algumas acontecem fora do controle da firma, algumas são coletivas (e carregam os problemas de coordenação e freeriding típicos deste tipo de ação), existem papéis que são mutáveis ao longo do processo. Além disso, estas são ações de não mercado (Baron, 1995), e, portanto, o gestor não tem bases de preços de mercado para avaliar seus investimentos. Esta característica do processo de estratégias políticas também afeta as questões de utilização de processos externos à firma e das ações coletivas. Esta característica do processo de estratégias políticas parece estar "sub-representada" na linha de pesquisa (Hillman et al., 2004). 


\section{Conclusões}

O governo tem enorme influência no desempenho das firmas (Ring, Bigley, D’Aunno, \& Khanna, 2005). As organizações podem elaborar, implementar e controlar estratégias políticas para proativamente influenciar o sistema político em busca de desempenho ou até mesmo sobrevivência. Apesar da importância dessas atividades, muito pouco se sabe sobre o processo dessas decisões estratégicas em si (Lux et al., 2011).

Por meio de uma pesquisa qualitativa usando entrevistas com 15 executivos seniores do setor e dados secundários, este artigo traz à tona algumas características particulares deste processo. Enquanto o processo de estratégias políticas assume muitas características semelhantes ao processo estratégico tradicional, as diferenças entre estes processos são a contribuição do artigo.

Tais diferenças se organizam em três fontes. A primeira delas consiste na existência de grandes porções do processo de estratégias políticas que se concentram fora do ambiente da empresa. Durante as discussões deste artigo, diversos grupos defenderam que a causa desta diferença poderia estar ligada à aura maldita que o lobbying e outras estratégias políticas têm (Galan, 2012), não só no Brasil, mas em todo o mundo. Embora seja uma possibilidade, as evidências não se mostraram conclusivas nesta direção. Possivelmente, seja uma receita de como fazer estratégia que tenha se desenvolvido entre os profissionais de relações governamentais (Porac et al., 2011).

Uma segunda fonte de diferenças está no papel predominante das formas coletivas de organização como forma de organização para o processo das estratégias políticas. É até surpreendente como são poucos os trabalhos que se aventuram em entender as minúcias deste processo, uma vez que as evidências mostram as dificuldades dos gestores de entenderem a dinâmica do que acontece em uma associação. Os jogos de desempenho de papéis (role -playing) já haviam sido reportados no passado (Cook \& Barry, 1995) e parecem bem presentes nas evidências deste estudo.

Finalmente, a terceira fonte de diferenças, que está intimamente ligada às anteriores, é a dificuldade na apuração dos resultados das estratégias políticas. Parece difícil entender o desenrolar de uma estratégia política (Mancuso, 2004), sobretudo quando existem diversos jogadores com interesses distintos, quando demandadores de políticas públicas e fornecedores de políticas públicas atuam em diversos papéis e quando os benefícios dessas podem ser assimétricos para as diferentes empresas envolvidas em particular setor. Este é um ponto, portanto, que aumenta a complexidade de um processo já cercado de incertezas (Dahan, 2009).

Esta pesquisa traz também suas limitações. Em razão de ser uma pesquisa qualitativa, deve-se tomar cuidado com a generalização dos achados. A amostra de entrevistados pode ter vieses relacionados, por exemplo, ao tamanho das empresas ou, ainda, às interações que ocorreram no passado entre estes atores. Também é possível que estas características do processo de estratégias políticas sejam particulares do setor de produção de etanol brasileiro.

Por conseguinte, sugere-se que futuras pesquisas em estratégias políticas foquem as questões micro deste processo. Os artigos seminais em estratégias políticas (Shaffer, 1995), há quase vinte anos, já apontavam para o desequilíbrio em favor de pesquisas com unidades de análise agregadas. O resultado deste desequilíbrio está no pequeno número de estudos que se centrem no trabalho dos gestores de relações governamentais. Também chama a atenção a quantidade limitada de trabalhos que evidenciem as questões das estratégias políticas coletivas de um ponto de vista da teoria organizacional e da estratégia, em contraponto aos trabalhos advindos das ciências políticas. Estas futuras pesquisas poderiam se valer do extenso material voltado à cooperação em estratégia e utilizá-lo para melhor entender as estratégias políticas. 


\section{Referências}

Amatucci, M., \& Spers, E. E. (2010). The Brazilian biofuel alternative. International Journal of Automotive Technology and Management, 10(1), 37-55. doi: 10.1504/IJATM.2010.031455

Ansolabehere, S., Figueiredo, J. M. de, \& Snyder, J. M. (2003). Why is there so little money in politics? Journal of Economic Perspectives, 17(1), 105-130. doi: 10.1257/089533003321164976

As Claras 2012, Transparência Brasil. (n.d.). Quem financia quem nas eleições. Recuperado de: http://www.asclaras.org.br/

Bandeira-de-Mello, R., \& Marcon, R. (2005). Unpacking firm effects: modeling political alliances in variance decomposition of firm performance in turbulent environments [1 ${ }^{\mathrm{a}}$ Edição Especial]. Revista de Administração Contemporânea, 9, 41-44. doi: 10.1590/S1415-65552005000500003

Bandeira-de-Mello, R., Marcon, R., \& Alberton, A. (2008). Drivers of discretionary firm donations in Brazil. Brazilian Administration Review, 5(4), 275-288. Recuperado de http://www.scielo.br/pdf/bar/v5n4/v5n4a03.pdf. 10.1590/S1807-76922008000400003

Baron, D. P. (1995). Integrated strategy: market and nonmarket components. California Management Review, 37(2), 47-65. doi: 10.2307/41165788

Bauer, M. W. (2000). Classic content analysis: a review. In M. W. Bauer \& G. D. Gaskell (Eds.), Qualitative researching with text, image and sound: a practical handbook for social research (pp. 131-151). Thousand Oaks: Sage Publications.

Bonardi, J.-P., Hillman, A. J., \& Keim, G. D. (2005). The attractiveness of political markets: implications for firm strategy. Academy of Management Review, 30(2), 397-413. doi: 10.5465/AMR.2005.16387895

Bonardi, J.-P., \& Keim, G. D. (2005). Corporate political strategies for widely salient issues. Academy of Management Review, 30(3), 555-576. doi: 10.5465/AMR.2005.17293705

Brey, N. K., Camilo, S. P. O., Marcon, R., \& Bandeira-de-Mello, R. (2013). Conexões políticas em estruturas de propriedade: o governo como acionista em uma análise descritiva. Revista de Administração Mackenzie, 15(1), 98-124. doi: 10.1590/S1678-69712014000100005

Burgelman, R. A. (1983). A process model of internal corporate venturing in the diversified major firm. Administrative Science Quarterly, 28(2), 223-244. doi: 10.2307/2392619

Caldeira, C. A. (2012). Aspectos cognitivos da formulação de estratégias políticas: um estudo de caso no setor de etanol brasileiro (Dissertação de mestrado). Fundação Getulio Vargas, Escola de Administração de Empresas, São Paulo, SP, Brasil.

Conejero, M. A. (2011). Planejamento e gestão estratégica de associações de interesse privado do agronegócio: uma contribuição empirica (Tese de doutorado). Universidade de São Paulo, São Paulo, SP, Brasil.

Cook, R. G., \& Barry, D. (1995). Shaping the external environment. Business \& Society, 34(3), 317344. doi: $10.1177 / 000765039503400304$

Cooper, D., \& Schindler, P. S. (2003). Métodos de pesquisa em administração. Porto Alegre: Bookman.

Costa, M., Bandeira-de-Mello, R., \& Marcon, R. (2013). Influência da conexão política na diversificação dos grupos empresariais brasileiros. Revista de Administração de Empresas, 53(4), 376-387. doi: 10.1590/S0034-75902013000400005 
Dahan, N. (2009). The four Ps of corporate political activity: a framework for environmental analysis and corporate action. Journal of Public Affairs, 9(2), 111-123. doi: 10.1002/pa.321

Doh, J., Lawton, T., \& Rajwani, T. (2012). Advancing nonmarket strategy research: institutional perspectives in a changing world. Academy of Management Perspectives, 26(3), 22-39. doi: 10.5465/amp.2012.0041

Doz, Y. L. (1996). The evolution of cooperation in strategic alliances: initial conditions or learning processes? Strategic Management Journal, 17(S1), 55-83. doi: 10.1002/smj.4250171006

Eisenhardt, K. M. (1989). Building theories from case study research. Academy of Management Review, 14(4), 532-550. doi: 10.5465/AMR.1989.4308385

Emerson, R. M., Fretz, R. I., \& Shaw, L. L. (1995). Writing ethnographic fieldnotes. Chicago: University of Chicago Press. doi: 10.7208/chicago/9780226206851.001.0001

Fernandes, C. M. A., Bandeira-de-Mello, R., \& Zanni, P. P. (2012). O papel dos fatores políticos na internacionalização de empresas: o caso da Energias de Portugal (EDP) no Brasil. Cadernos EBAPE.BR, 10(2), 435-455. Recuperado de http://www.scielo.br/pdf/cebape/v10n2/v10n2a11.pdf. doi: 10.1590/S1679-39512012000200011

Freitas, H., \& Janissek, R. (2000). Análise léxica e análise de conteúdo: técnicas complementares, seqüenciais e recorrentes para exploração de dados qualitativos. Porto Alegre: Sagra Luzzatto.

Freitas, J. (2011, abril 9). Intervenção nos combustíveis. Correio Brasiliense - DF. Universidade de Brasília. Recuperado de http://www.unb.br/noticias/unbagencia/cpmod.php?id=87820

Galan, G. (2012). Relações governamentais \& lobby: aprendendo a fazer. São Paulo: Aberje.

Getz, K. A. (1997). Research in corporate political action. Business \& Society, 36(1), 32-72. doi: $10.1177 / 000765039703600103$

Gulati, R., Lavie, D., \& Singh, H. (2009). The nature of partnering experience and the gains from alliances. Strategic Management Journal, 30(11), 1213-1233. doi: 10.1002/smj.786

Hadani, M., \& Schuler, D. A. (2013). In search of El Dorado: the elusive financial returns on corporate political investments. Strategic Management Journal, 34(2), 165-181. doi: 10.1002/smj.2006

Hamel, G. (1991). Competition for competence and interpartner learning within international strategic alliances. Strategic Management Journal, 12(S1), 83-103. doi: 10.1002/smj.4250120908

Hillman, A. J., \& Hitt, M. A. (1999). Corporate political strategy formulation: a model of approach, participation, and strategy decisions. Academy of Management Review, 24(4), 825-842. doi: 10.5465/AMR.1999.2553256

Hillman, A. J., Schuler, D. A., \& Keim, G. D. (2004). Corporate political activity: a review and research agenda. Journal of Management, 30(6), 837-857. doi: 10.1016/j.jm.2004.06.003

Hutzschenreuter, T., \& Kleindienst, I. (2006). Strategy-process research: what have we learned and what is still to be explored. Journal of Management, 32(5), 673-720. doi: 10.1177/0149206306291485

Jornal Cana. (2007, março 22). Alcopar quer construção de duto entre Maringá e o Porto de Paranaguá. Recuperado de http://www.jornalcana.com.br/alcopar-quer-construcao-de-duto-entre-maringa-eo-porto-de-paranagua/

Lazzarini, S. (2011). Capitalismo de laços. São Paulo: Campus.

Leão, J. (2002). Álcool energia verde. São Paulo: Iqual. 
Lux, S., Crook, T. R., \& Woehr, D. J. (2011). Mixing business with politics: a meta-analysis of the antecedents and outcomes of corporate political activity. Journal of Management, 37(1), 223-247. doi: $10.1177 / 0149206310392233$

Mancuso, W. P. (2004). O lobby da indústria no Congresso Nacional: empresariado e política no Brasil contemporâneo. Dados, 47(3), 505-547. doi: 10.1590/S0011-52582004000300003

Merriam, S. B. (1988). Case study research in education: a qualitative approach. San Francisco, CA: Jossey-Bass.

Mintzberg, H. (1978). Patterns in strategy formation. Management Science, 24(9), 934-948. doi: 10.1287/mnsc.24.9.934

Mintzberg, H., \& Quinn, J. B. (1998). Readings in the strategy process. Upper Saddle River, NJ: Prentice Hall.

Neves, M. F., \& Conejero, M. (2010). Estratégias para a cana no Brasil: um negócio classe mundial. São Paulo: Atlas.

Nielsen, R. P. (1988). Cooperative strategy. Strategic Management Journal, 9(5), 475-492. doi: 10.1002/smj.4250090507

Noda, T., \& Bower, J. L. (1996). Strategy making as iterated processes of resource allocation. Strategic Management Journal, 17(S1), 159-192. doi: 10.1002/smj.4250171011

Olson, M. (1971). The logic of collective action: public goods and the theory of groups (Vol. 124). Cambridge: Harvard University Press.

Pereira, R., \& Chade, J. (2012, janeiro 16). Usineiros buscam prestígios perdidos. O Estado de São Paulo. Recuperado de http://www.estadao.com.br/noticias/impresso, usineiros-buscamprestigios-perdidos, $823142,0 . \mathrm{htm}$

Pettigrew, A. M. (1992). The character and significance of strategy process research. Strategic Management Journal, 13(S2), 5-16. doi: 10.1002/smj.4250130903

Porac, J. F., Thomas, H., \& Baden-Fuller, C. (2011). Competitive groups as cognitive communities: the case of scottish knitwear manufacturers revisited. Journal of Management Studies, 48(3), 646664. doi: 10.1111/j.1467-6486.2010.00988.x

Prashant, K., \& Harbir, S. (2009). Managing strategic alliances: what do we know now, and where do we go from here? The Academy of Management Perspectives, 23(3), 45-62. doi: 10.5465/AMP.2009.43479263

Richardson, R. J. (2009). Pesquisa social métodos e técnicas. São Paulo: Atlas.

Ring, P. S., Bigley, G. A., D'Aunno, T., \& Khanna, T. (2005). Introduction to special topic forum: perspectives on how goverments matter. Academy of Management Review, 30(2), 308-320. doi: 10.5465/AMR.2005.16387887

Samuels, D. (2001). Incumbents and challengers on a level playing field: assessing the impact of campaign finance in Brazil. The Journal of Politics, 63(2), 569-584. doi: 10.1111/00223816.00079

Schneider, B. R. (2010). Business politics in Latin America: patterns of fragmentation. In D. Coen, W. Grant, \& G. Wilson (Eds.), The Oxford handbook of business and government (pp. 307-329). Oxford: Oxford University Press. 
Selltiz, C., Wrightsman, L. S., \& Cook, S. W. (1976). Research methods in social research. New York: Holt, Rinehart \& Winston.

Shaffer, B. (1995). Firm-level responses to government regulation: theoretical and research approaches. Journal of Management, 21(3), 495-514. doi: 10.1177/014920639502100305

Shaffer, B., \& Hillman, A. J. (2000). The development of business-government strategies by diversified firms. Strategic Management Journal, 21(2), 175-190. doi: 10.1002/(SICI)10970266(200002)21:2<175::AID-SMJ86>3.0.CO;2-L

Siggelkow, N. (2007). Persuasion with case studies. Academy of Management Journal, 50(1), 20-24. doi: 10.5465/AMJ.2007.24160882

Szmrecsanyi, T. M., \& Moreira, E. P. (1991). O desenvolvimento da agroindústria canavieira do Brasil desde a segunda guerra mundial. Estudos Avançados, 5(11), 57-79. doi: 10.1590/S010340141991000100006

Van De Ven, A. H. (1992). Suggestions for studying strategy process: a research note. Strategic Management Journal, 13(S1), 169-188. doi: 10.1002/smj.4250131013

Yin, R. (2008). Case study research: design and methods. Thousand Oaks: Sage Publications. 